\title{
Comparison of Radiographic, Ultrasound, and Magnetic Resonance Imaging for the Detection of Retained Stingray Barb: A Cadaveric Study
}

\author{
Taylor A. Docter, $\mathrm{MD}^{1}$; Lauren B. Altschuh, $\mathrm{MD}^{2}$; Anthony J. Medak, $\mathrm{MD}^{2}$; Sheronda M. Statum, $\mathrm{PhD}^{3}$; \\ Christine B. Chung, $\mathrm{MD}^{3}$; Karen B. Van Hoesen, $\mathrm{MD}^{2}$; Christanne H. Coffey, MD ${ }^{2}$ \\ ${ }^{1}$ Department of Emergency Medicine, LAC+USC Medical Center, Los Angeles, California; ${ }^{2}$ Department of Emergency Medicine, University of \\ California San Diego, San Diego, California; ${ }^{3}$ Department of Radiology, University of California San Diego, San Diego, California
}

Introduction-Stingray envenomations are a common marine animal injury for which it is important to identify and remove retained barbs to prevent secondary infection. The optimal imaging modality in stingray foreign body detection is not well characterized in the existing literature. In this study, we compared the accuracy of plain radiography, ultrasound, and magnetic resonance imaging (MRI) in detecting stingray barbs in the human foot and ankle.

Methods-This cadaveric study included a 1:1 randomization to the presence or absence of barbs in 24 sample injuries of human cadaveric foot and ankle specimens. Physicians trained in emergency medicine and radiology performed ultrasound examinations on each specimen and interpreted the presence or absence of a barb. Participants also interpreted x-ray images in the same manner. MRI scans were separately interpreted by a musculoskeletal radiology attending. Data were analyzed using McNemar's test.

Results-The 19 participants included $14(74 \%)$ trained in emergency medicine and $5(26 \%)$ trained in radiology. Forty-seven percent were residents, $42 \%$ faculty, and $11 \%$ fellows. X-ray was associated with the highest sensitivity of $94 \%$ for the identification of a retained barb, followed by MRI (83\%) and ultrasound (70\%). MRI was associated with the highest specificity of $100 \%$, followed by x-ray $(98 \%)$ and ultrasound $(73 \%)$.

Conclusions-Retained stingray barbs can lead to secondary infection after envenomation. In human cadaveric specimens, $\mathrm{x}$-ray demonstrated the highest sensitivity, MRI demonstrated the highest specificity, and ultrasound demonstrated lower sensitivity and specificity.

Keywords: envenomation, x-ray, ultrasonography, foreign body, spine, ray

\section{Introduction}

With over 150 different species existing in both seawater and freshwater, the stingray is often sought out as an underwater attraction for snorkelers and scuba divers. ${ }^{1}$ However, armed with 1 to 4 retroserrated barbs with associated venom glands on its tail, the stingray is prepared to attack when threatened. ${ }^{2}$ Anywhere from 750 to 2000 stingray injuries are reported in the United States

Corresponding author: Christanne H. Coffey, MD, UC San Diego Health, 200 W. Arbor Drive \#8676, San Diego, CA 92103; e-mail: chcoffey@health.ucsd.edu.

Submitted for publication August 2020.

Accepted for publication March 2021. each year, ${ }^{3,4}$ making them a commonly reported marine animal to cause human envenomation. The wound inflicted is typically a combination of a puncture wound and laceration and occurs most commonly on the lower extremity. ${ }^{5}$ As the barbed tail contacts the swimmer's skin, the integumentary sheath that covers the barb is torn, releasing venom into the wound, and fragments of both the sheath and the barb may be retained at the site of injury. ${ }^{1}$ Although most injuries do not jeopardize the life of a patient, the released venom causes immediate pain and vasoconstriction, and some patients may develop systemic symptoms including but not limited to nausea, vomiting, diarrhea, muscle cramps, hypotension, and cardiac dysrhythmias. ${ }^{1,5}$ Retention of stingray barbs has 
the potential to lead to secondary infection, which in some rare cases has progressed to life-threatening necrotizing fasciitis. ${ }^{6,7}$ For this reason, it is important to identify and remove any retained foreign bodies from a stingray injury. ${ }^{1,8}$

Though literature on management of stingray envenomation is limited, hot water immersion is the mainstay of treatment. ${ }^{6}$ Antibiotics and pain medications may also be used. Many individuals have adequate control of symptoms after hot water immersion ${ }^{9-11}$ on the beach or at home and require no further evaluation or treatment. Those stingray injuries that require further medical attention are typically evaluated in an emergency department, urgent care, or other primary care setting where x-ray imaging and bedside ultrasound may be available; however, magnetic resonance imaging (MRI) may not be accessible. It is generally recommended that if a retained foreign body is suspected, wounds should be explored and debrided to ensure all remnants of the barb and sheath are removed to prevent delayed healing and infection. ${ }^{12}$ The utility of radiographs, ultrasound, and MRI to aid in this endeavor has yet to be well characterized in the literature. ${ }^{4}$ The value of radiographs, specifically, has been a long debated topic in the management of these injuries, as some sources suggest that stingray barbs may not be radiopaque whereas other case reports indicate successful visualization with $\mathrm{x}$-rays. ${ }^{4,13}$ To our knowledge, the use of ultrasound and MRI as potential imaging modalities in detecting retained barbs has not been studied. The aim of our study was to compare the accuracy of plain radiography, ultrasound, and MRI in detecting retained stingray barbs in the human foot and ankle.

\section{Methods}

This study was submitted to the University of California San Diego institutional review board and deemed exempt. It was approved by the Birch Aquarium at Scripps animal care use committee.

In this cadaveric study, we randomized the presence or absence of stingray barbs in predetermined locations of 12 human fresh frozen cadaver foot and ankle specimens. The foot and lower ankle were chosen as the anatomic sites for this study because they are the most common locations of injury. Round stingray (Urobatis halleri) barbs were collected over a 6-mo period when trimming was performed by Birch Aquarium staff as part of their routine animal management protocols.

We obtained 12 fresh frozen cadaveric specimens and used 2 sites (foot and ankle) per specimen to mimic the stingray injury, providing a total of 24 samples for interpretation. There were 4 samples for each of the 6 anatomic sites: the medial arch, lateral arch, and heel on the foot and the medial malleolus, lateral malleolus, and posterior calf on the ankle. We replicated a small skin defect for each simulated injury, mimicking the common clinical presentation of a stingray puncture wound. We made a 5-mm incision at a $30^{\circ}$ angle using an 11-blade scalpel and then inserted and opened a hemostat to $20^{\circ}$ to provide the same tissue distortion for each sample site. The injury sites were randomized in a 1:1 fashion for the presence or absence of a retained barb. In those sites randomized to include the presence of a barb, a standard 3-mm by $15-\mathrm{mm}$ piece of stingray barb was inserted into the simulated wound defect using a hemostat until the barb was no longer visualized at the wound defect or palpable. The fragment was the same width as and approximately half the length of the round stingray barbs collected. This fragment size was chosen because it was believed to be similar in dimension to other retained organic materials, such as cactus spines or large wooden splinters.

Radiographs were performed and recorded by trained technicians for each cadaveric specimen. A radiopaque marker was placed over the skin defect, and anteroposterior and lateral radiographs of the foot and the ankle were obtained using the Siemens Ysio Max machine. These images were de-identified and randomized in order. The radiopaque markers were removed, and the site of injury on each specimen was marked with a skin marker before the ultrasound and MRI portions of the study. Specimens were again de-identified and randomized for the ultrasound and MRI interpretation.

Emergency medicine (EM) and radiology physician trainees and attendings were recruited to participate as evaluators in the study. Participants filled in a written questionnaire for demographic data, including medical specialty, training level, and any additional pertinent training or certification in ultrasound or musculoskeletal imaging. Participants were blinded to which extremity sites actually had a retained foreign body. EM physicians attended a 20-min didactic session in which principles of soft tissue ultrasound and foreign body location were discussed, including proper scanning technique and methods of visualization. In addition, all EM resident physicians at the study location had baseline ultrasound training that included over $20 \mathrm{~h}$ of didactic and hands-on scanning during the postgraduate year (PGY)-1, which included soft tissue applications. After the ultrasound didactic presentation, all EM and radiology physicians used a high frequency linear (HFL50x) transducer on the Sonosite Edge II ultrasound system to review the cadaveric specimens and record whether a foreign body was present. Participants worked independently and 


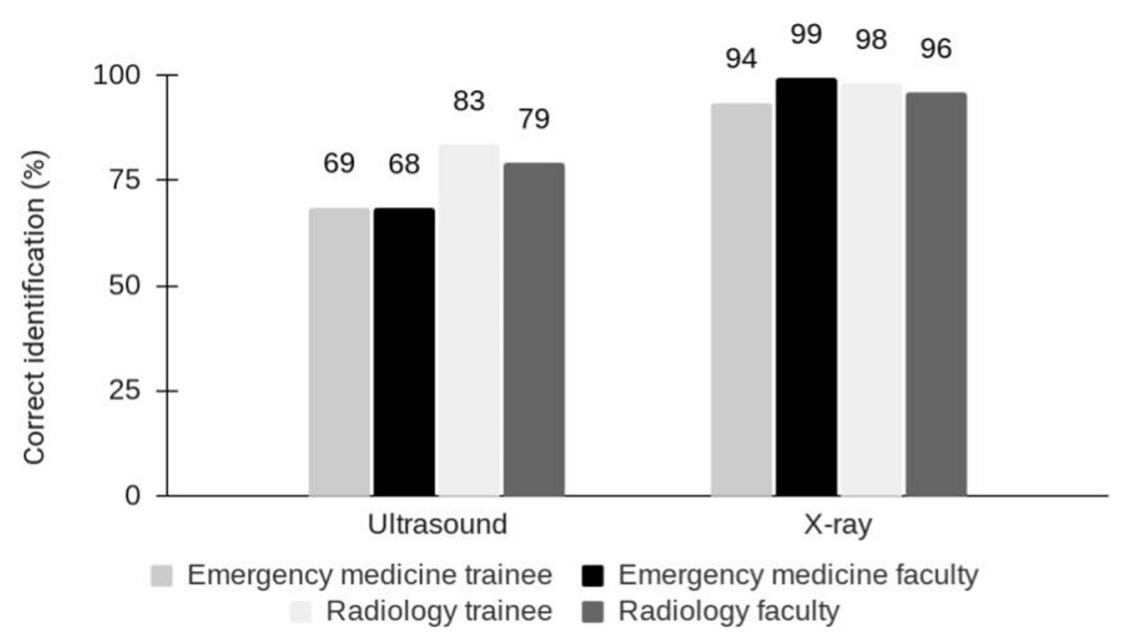

Figure 1. Identification of retained stingray barb by imaging modality, level of training, and specialty.

without a time limit. Commercial ultrasound gel and gelfilled gloves, used as a standoff pad, were provided for the ultrasound scanning portion. The x-rays of the same specimens were then reviewed by the same physicians, who were blinded as to which extremities had a retained foreign body. The reviewers recorded whether a foreign body was detected.

Standard MRI sequences were acquired in routine imaging planes, again randomized in order and then reviewed by 1 board certified musculoskeletal attending radiologist, who was blinded as to which extremities had a retained foreign body. The reviewer recorded whether a foreign body was detected on MRI.

\section{STATISTICS}

For each imaging technique (x-ray, ultrasound, and MRI), we calculated the overall detection sensitivity, specificity, false positive rate, and false negative rate along with individual reviewers' sensitivity and specificity. The test characteristics were compared among imaging modalities using McNemar's test. Interobserver reliability was compared using the Fleiss kappa test. Comparing sensitivity and specificity of the various imaging methods, with alpha set at 0.05 and a sample size of over 430 ( 24 specimen "injuries" $\times 18$ participants), the study had an $80 \%$ power to detect an effect size of $15 \%$.

\section{Results}

The 19 reviewers included 14 (74\%) emergency physicians and $5(26 \%)$ radiologists. The emergency physicians included $9(64 \%)$ resident physicians and $5(36 \%)$ attending physicians. The EM resident physician PGY breakdown was 3 (33\%) PGY-1, 5 (56\%) PGY-2, and 1 (11\%) PGY-4. Among the EM attending physicians, 1 (20\%) was a registered diagnostic medical sonographer, 2 (40\%) had completed ultrasound fellowships, and 3 $(60 \%)$ were ultrasound credentialed within the EM department. All EM attendings were board certified in EM. The breakdown of radiologists included $2(40 \%)$ musculoskeletal fellow physicians and $3(60 \%)$ attending physicians board certified in radiology.

$\mathrm{X}$-ray was associated with the highest sensitivity of $94 \%$ for the identification of a retained barb, followed by MRI (83\%) and ultrasound (70\%). MRI was associated with the highest specificity of $100 \%$, followed by x-ray (98\%) and ultrasound (73\%).

There was no difference in correctly identifying the presence or absence of a retained barb on ultrasound or X-ray based on the reviewer's level of training ( $P=0.13$ and $P=0.85$, respectively) (Figure 1 ). There was no difference observed amongst PGY training for the EM resident physicians. For X-ray, success rates ranged from 94 to $99 \%$. There was no difference in the accuracy of identification of a stingray barb on $x$-ray between the EM physicians and the radiology physicians $(P=0.68)$. For ultrasound, success rates ranged from 68 to $83 \%$ with radiology faculty and fellows performing better than their EM colleagues $(P=0.03)$.

Foreign body identification accuracy was also evaluated based on the anatomic location of injury by imaging modality (Figure 2). The easiest location for identification was the heel, at $87 \%$ sensitivity for ultrasound and $100 \%$ sensitivity for both $\mathrm{x}$-ray and MRI. The medial arch proved most challenging for $\mathrm{x}$-ray ( $82 \%$ sensitivity), whereas the medial and lateral malleoli were the most challenging for MRI ( $75 \%$ sensitivity). The remainder of 


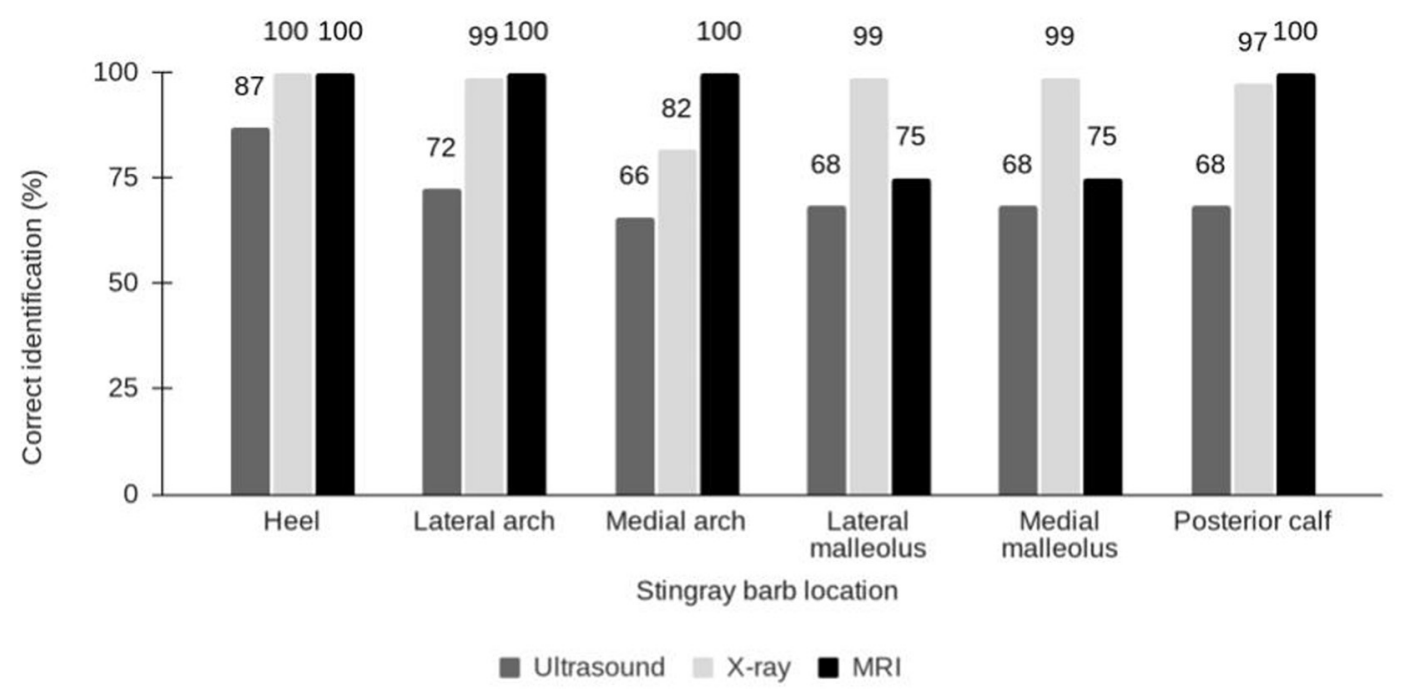

Figure 2. Identification of retained stingray barb by anatomic location and imaging modality.

locations did not show a difference in accuracy in regard to ultrasound imaging.

\section{Discussion}

This cadaveric study found that plain radiography was associated with the highest sensitivity for detection of retained stingray barb after injury and that MRI was associated with the highest specificity. Ultrasound was associated with the lowest sensitivity and specificity of the 3 modalities studied.

Radiology physicians performed better than EM physicians in identifying retained barbs on ultrasound. One of the radiology faculty and both radiology fellows had special training in musculoskeletal imaging, which may have contributed to their better performance on ultrasound identification of barb retention. Overall, variations in performance across different levels of training and specialties speak to user discrepancy in interpreting both ultrasound and x-ray images. Furthermore, when using ultrasound, it is important to consider variability in user proficiency in obtaining adequate images, in addition to their ability to interpret those images.

The heel was the anatomic site associated with the highest rate of accuracy across all 3 imaging modalities (Figure 3). For MRI, injuries that were simulated at the medial malleolus and lateral malleolus had the lowest percentage of correctly identified images. We suspect this observation is related to the type of surrounding tissue at each site. The heel contains a subcutaneous fat pad that assists in providing contrast for stingray barb identification, whereas the malleoli are bony structures with less surrounding subcutaneous tissue and therefore provide less contrast for barb identification.

\section{LIMITATIONS}

This study has limitations that are inherent to a cadaveric study. The use of cadaveric specimens and simulated injury does not provide the exact environment that would be encountered in the clinical setting. Soft tissue swelling is often present in stingray injuries, and fluid collections may be seen when a deep space infection has developed. These associated findings may enhance visualization via ultrasound or MRI, which use variations in tissue density to identify structures.

Frequently a water bath is used in the clinical setting to enhance ultrasound images when searching for retained foreign bodies. We decided not to include a water bath as an option, given the potential risk of water dissecting down into the simulated wounds and distorting images for subsequent evaluators. Additionally, there was concern for loss of integrity of the cadaveric specimens if they were submerged in water repeatedly. Gel-filled gloves, to be used as standoff pads, were offered as an alternative to commercial ultrasound gel; however, the gloves were infrequently used by the participants. Using water baths may have increased the accuracy of ultrasound identification. ${ }^{14}$

It is notable that we had clinician-sonographers, including PGY-1 physicians, obtaining and interpreting the ultrasound images, whereas the MRI scans were obtained by MRI technologists and interpreted by a board-certified musculoskeletal radiologist. Given the vast difference in training and expertise of our 



Figure 3. The heel was the anatomic site associated with the highest rate of accuracy across all 3 imaging modalities. A, Lateral view $x$-ray demonstrating stingray barb (arrow). B, Sagittal cut of magnetic resonance imaging demonstrating stingray barb (arrow). C, Ultrasound demonstrating stingray barb (arrow) in the longitudinal axis.

physician participants, our results do not provide a head-to-head comparison of ultrasonography and MRI in the detection of stingray barbs in human feet and ankles. The possibility of user error in ultrasound image acquisition and interpretation presents a challenge in determining whether ultrasonography is truly inferior. Rather, our study sought to assess the relative merits of provider-performed ultrasound (point-of-care ultrasound) compared with radiology-performed imaging modalities. In our urban, academic emergency department setting, it is commonplace for providers to perform such focused ultrasound examinations at the bedside. However, given that this may not be the norm in other practice settings, our results are less generalizable.

The small number of participants from different specialties and training levels also limits any conclusions regarding the optimal physician background in performing these imaging studies. Moreover, there was only a single MRI reviewer, compared to multiple reviewers for ultrasound and plain radiography.

Finally, our study used stingray barbs that had been stored and no longer had an integumentary sheath, which typically encases the barb in live animals. In some clinical cases, fragments of the integumentary sheath may be left in the wound without any barb fragments, and this organic material can also be a nidus of infection, leading to antibiotic use or potential foreign body removal. We did not use freshly trimmed barbs in this investigation because it took several months to collect an adequate number of barbs to complete the study. Our study instead focused exclusively on the comparison of imaging modalities in identifying barb fragments rather than retained portions of the integumentary sheath. If feasible, future investigations of the integumentary sheath alone or using freshly trimmed barbs could yield different results than the current study. This research used barbs from round stingrays. It is unknown whether the species of stingray affects how barbs 
are visualized using different imaging modalities. Studies using other stingray species may have different results.

\section{Conclusions}

Retention of stingray barbs has the potential to turn a low-risk injury into a life-threatening infection. In human cadaveric specimens, x-ray demonstrated the highest sensitivity, MRI demonstrated the highest specificity, and clinician-performed ultrasound demonstrated the lowest sensitivity and specificity in detecting retained stingray barbs. In this study, EM physicians had the same accuracy in identifying stingray barbs on plain radiographs as radiology physicians. Given that plain radiography demonstrated higher sensitivity than MRI in our study, and that MRI is more time-consuming and costly, we would recommend considering the use of plain radiography as the initial imaging modality to look for retained barb fragments after a stingray injury.

Acknowledgments: The authors acknowledge the individuals who have donated their bodies to science and make research like this possible. The authors thank the Birch Aquarium at Scripps Institution of Oceanography for providing the stingray barbs used in this research. The authors thank Dr. Sukhdeep Singh and Dr. Kaitlin McIntyre for their contribution to this research, and they thank the emergency medicine and radiology physicians who participated in this study.

This work was presented as an oral abstract at the Wilderness Medical Society Summer Virtual Conference, July 22, 2020.

Author Contributions: Study concept and design (CHC, TD, KVH, SMS, AM, CBC); acquisition of the data (TD, LA, KVH, AM, SMS, $\mathrm{CBC}, \mathrm{CHC}$ ); analysis and interpretation of the data (LA, TD, $\mathrm{CHC}$ ); drafting of the manuscript (TD, CHC, LA); critical revision of the manuscript (CHC, KVH, AM, SMS, CBC). All authors approved the final manuscript.

Financial/Material Support: This study received funding from the Wilderness Medical Society Houston Grant, supported by the Academy of Wilderness Medicine ${ }^{\circledR}$. The Sonosite Edge II ultrasound systems were provided for the research day from FUJIFILM Sonosite, Inc. MRI and interpretation were provided by the University of California San Diego MSK MRI Research Lab.

Disclosures: None.

\section{References}

1. Fernandez I, Valladolid G, Varon J, Sternbach G. Encounters with venomous sea-life. J Emerg Med. 2011;40(1):103-12.

2. Jarvis HC, Matheny LM, Clanton TO. Stingray injury to the webspace of the foot. Orthopedics. 2012;35(5):e762-5.

3. Diaz JH. The evaluation, management, and prevention of stingray injuries in travelers. J Travel Med. 2008; 15(2):102-9.

4. Clark RF, Girard RH, Rao D, Ly BT, Davis DP. Stingray envenomation: a retrospective review of clinical presentation and treatment in 119 Cases. J Emerg Med. 2007;33(1):33-7.

5. Evans RJ, Davies RS. Stingray injury. J Accid Emerg Med. 1996;13(3):224-5.

6. Myatt T, Nguyen B, Clark R, Coffey C, O'Connell C. A prospective study of stingray injury and envenomation outcomes. J Emerg Med. 2018;55(2):213-7.

7. Barber GR, Swygert JS. Necrotizing fasciitis due to Photobacterium damsela in a man lashed by a stingray. $N$ Engl J Med. 2000;342(11):824.

8. Diaz JH. The epidemiology, evaluation, and management of stingray injuries. J La State Med Soc. 2007;159(4):198-204.

9. Russell FE, Panos TC, Kang LW, Warner AM, Colket 3rd TC. Studies on the mechanism of death from stingray venom; a report of two fatal cases. Am J Med Sci. 1958;235(5):566-84.

10. Clark AT, Clark RF, Cantrell FL. A retrospective review of the presentation and treatment of stingray stings reported to a poison control system. Am $J$ Ther. 2017;24(2):e177-80.

11. Isbister GK. Venomous fish stings in tropical northern Australia. Am J Emerg Med. 2001;19(7):561-5.

12. Bendt RR, Auerbach PS. Foreign body reaction following stingray envenomation. J Wilderness Med. 1991;2(4):298-303.

13. Srinivasan S, Bosco JI, Lohan R. Marine stingray injuries to the extremities: series of three cases with emphasis on imaging. J Postgrad Med. 2013;59(4):309-11.

14. Krishnamurthy R, Yoo JH, Thapa M, Callahan MJ. Waterbath method for sonographic evaluation of superficial structures of the extremities in children. Pediatr Radiol. 2013;43(Suppl 1):S41-7. 